\title{
Relevance of the incubation period in cytotoxicity testing with primary human hepatocytes
}

\author{
Xiaolong Gu ${ }^{1,2} \cdot$ Wiebke Albrecht ${ }^{1} \cdot$ Karolina Edlund $^{1}$ • Franziska Kappenberg ${ }^{3}$. Jörg Rahnenführer ${ }^{3}$. \\ Marcel Leist ${ }^{4} \cdot$ Wolfgang Moritz ${ }^{5}$. Patricio Godoy ${ }^{1} \cdot$ Cristina Cadenas $^{1} \cdot$ Rosemarie Marchan $^{1} \cdot$ Tim Brecklinghaus $^{1}$. \\ Laia Tolosa Pardo ${ }^{6} \cdot$ José V. Castell $^{6} \cdot$ Iain Gardner $^{7} \cdot$ Bo Han $^{2} \cdot$ Jan G. Hengstler $^{1} \cdot$ Regina Stoeber $^{1}$
}

\begin{abstract}
Primary human hepatocytes (PHHs) remain the gold standard for in vitro testing in the field of pharmacology and toxicology. One crucial parameter influencing the results of in vitro tests is the incubation period with test compounds. It has been suggested that longer incubation periods may be critical for the prediction of repeated dose toxicity. However, a study that systematically analyzes the relationship between incubation period and cytotoxicity in PHHs is not available. To close this gap, 30 compounds were tested in a concentration-dependent manner for cytotoxicity in cultivated cryopreserved PHHs (three donors per compound) for 1, 2 and 7 days. The median of the $\mathrm{EC}_{50}$ values of all compounds decreased 1.78-fold on day 2 compared to day 1, and 1.89-fold on day 7 compared to day 1 . Median values of $\mathrm{EC}_{50}$ ratios of all compounds at day 2 and day 7 were close to one but for individual compounds the ratio increased up to almost six. Strong correlations were obtained for $\mathrm{EC}_{50}$ on day 1 and day $7(R=0.985$; 95\% CI $0.960-0.994)$, day 1 and day $2(R=0.964 ; 95 \%$ CI $0.910-0.986)$, as well as day 2 and day $7(R=0.981 ; 95 \%$ CI 0.955-0.992). However, compound specific differences also occurred. Whereas, for example, busulfan showed a relatively strong increase on day 7 compared to day 1, cytotoxicity of acetaminophen did not increase during longer incubation periods. To validate the observed correlations, a publicly available data set, containing data on the cytotoxicity of human hepatocytes cultivated as spheroids for incubation periods of 5 and 14 days, was analyzed. A high correlation coefficient of $\mathrm{EC}_{50}$ values at day 5 and day 14 was obtained $(R=0.894 ; 95 \%$ CI $0.798-0.945)$. In conclusion, the median cytotoxicity of the test compounds increased between 1 and 2 days of incubation, with no or only a minimal further increase until day 7. It remains to be studied whether the different results obtained for some individual compounds after longer exposure periods would correspond better to human-repeated dose toxicity.
\end{abstract}

Keywords Hepatotoxicity · Primary human hepatocyte $\cdot$ Incubation period $\cdot$ Cell-titer-blue

Xiaolong Gu and Wiebke Albrecht shared first authorship.

Jan G. Hengstler

hengstler@ifado.de

1 Leibniz Research Centre for Working Environment and Human Factors at the Technical University of Dortmund (IfADo), 44139 Dortmund, Germany

2 College of Veterinary Medicine, Yunnan Agricultural University, Kunming 650201, Yunnan, People's Republic of China

3 Department of Statistics, TU Dortmund University, 44227 Dortmund, Germany

$\begin{array}{ll}\text { Abbreviations } \\ \text { APAP } & \text { Acetaminophen } \\ \text { ASP } & \text { Aspirin } \\ \text { BOS } & \text { Bosentan } \\ \text { BPR } & \text { Buspirone }\end{array}$

4 In Vitro Toxicology and Biomedicine, Department of Biology, University of Konstanz, Universitätsstr. 10, PO Box M657, 78457 Konstanz, Germany

5 InSphero AG, Technoparkstr. 1, 8005 Zürich, Switzerland

6 Unit for Cell Therapy, University La Fe Hospital, Valencia, Spain

7 Simcyp, Sheffield, UK 


$\begin{array}{ll}\text { BUSF } & \text { Busulfan } \\ \text { CBZ } & \text { Carbamazepine } \\ \text { CHL } & \text { Chlorpheniramine } \\ \text { CLON } & \text { Clonidine } \\ \text { DFN } & \text { Diclofenac } \\ \text { DMSO } & \text { Dimethyl sulfoxide } \\ \text { DILI } & \text { Drug-induced liver injury } \\ \text { EtOH } & \text { Ethanol } \\ \text { FAM } & \text { Famotidine } \\ \text { Glc } & \text { Glucose } \\ \text { HYZ } & \text { Hydroxyzine } \\ \text { INAH } & \text { Isoniazid } \\ \text { KC } & \text { Ketoconazole } \\ \text { LAB } & \text { Labetalol } \\ \text { LEV } & \text { Levofloxacin } \\ \text { MEL } & \text { Melatonin } \\ \text { MePa } & \text { Methylparaben } \\ \text { NAC } & \text { N-Acetylcysteine } \\ \text { NIM } & \text { Nimesulide } \\ \text { NFT } & \text { Nitrofurantoin } \\ \text { PhB } & \text { Phenylbutazone } \\ \text { PMZ } & \text { Promethazine } \\ \text { PPL } & \text { Propranolol } \\ \text { RIF } & \text { Rifampicin } \\ \text { TSN } & \text { Triclosan } \\ \text { VPA } & \text { Valproic acid } \\ \text { VitC } & \text { Vitan C }\end{array}$

Vit C Vitamin C

\section{Introduction}

Drug-induced liver injury (DILI) is one of the principal reasons for drug withdrawal from the market (Godoy et al. 2013; Hewitt et al. 2007). DILI also belongs to the most frequent causes of acute liver failure in industrialized countries (Bernal et al. 2010; Ostapowicz et al. 2002; Wilke et al. 2007), and occurs despite conducting standard preclinical tests, such as subchronic and chronic rodent studies.

Currently, primary human hepatocytes represent the gold standard model for in vitro testing of drug metabolism and cytotoxicity (LeCluyse 2001). For cytotoxicity testing, incubation periods of 1 or 2 days are usually used (Arbo et al. 2016; Ghallab et al. 2016); however, it has been reported that longer incubation periods may influence the test result (Proctor et al. 2017). A study that systematically analyzes the relationship between incubation period and cytotoxicity in primary human hepatocytes (PHHs) is not yet available. Therefore, we selected a set of 30 compounds, mostly pharmaceutical drugs, to perform concentration and time dependent incubations of cultivated hepatocytes. The aim of the present study was to compare cytotoxicity after 1,2 and 7 days of test compound exposure to understand, if EC50 values change depending on the length of the incubation period, and whether this change is by a similar factor for all compounds or if large compound specific differences occur. The relevance of the observed effects for toxicological routine testing will be discussed.

\section{Materials and methods}

\section{Chemicals and cells}

Williams medium E, penicillin/streptomycin solution, SeraPlus (FCS), and stable L-glutamine were purchased from PAN Biotech (Aidenbach, Germany), Gentamicin (10 mg/ $\mathrm{mL}$ ) was obtained from Invitrogen Corp. (Karlsruhe, Germany) and insulin supplement (ITS), dexamethasone, trypan blue solution, and all test compounds except ethanol were purchased from Sigma-Aldrich (St. Louis, USA). Ethanol was obtained from VWR chemicals (Mannheim, Germany), and rat-tail tendon collagen I for monolayer culture was obtained from Roche (Mannheim, Germany). Cell-TiterBlue Cell Viability assay was purchased from Promega (Mannheim, Germany), and cryopreserved primary human hepatocytes were obtained from BioreclamationIVT (Baltimore, USA) (details in Supplement 1).

\section{Cell culture}

Cryopreserved primary human hepatocytes (PHH) from three donors (AFJ, IAN and MSW) (BioreclamationIVT) were used. Cultivation of cryopreserved hepatocytes was performed according to a published standard operating procedure (SOP) (Godoy et al. 2013) with modifications. The SOP used for the current study is available in Supplement 2. Briefly, for the gel preparation, a bottle of $10 \mathrm{mg}$ lyophilized rat-tail collagen was dissolved overnight in $40 \mathrm{~mL} 0.2 \%$ acetic acid at $4{ }^{\circ} \mathrm{C}$. Each well of 96-well plates was coated with $100 \mu \mathrm{L}(250 \mu \mathrm{g} / \mathrm{mL})$ collagen solution. The collagen solution was removed immediately, and the plates were left to dry overnight under the cell culture hood. The plates coated with collagen were washed three times with sterile PBS. Cryopreserved primary human hepatocytes were thawed in a water bath $\left(37^{\circ} \mathrm{C}\right)$ and immediately transferred into a Falcon tube with culture medium containing 10\% FCS. After cell counting using Trypan blue to determine viability, 50,000 cells in FCS-containing medium were plated into each well of 96-well plates and kept at $37{ }^{\circ} \mathrm{C}$ for at least $3 \mathrm{~h}$. For homogenous distribution of the cells, the plate was gently shaken every 5-10 min during the first half hour of incubation. After the attachment period of $3 \mathrm{~h}$, the cells were washed with warm sterile PBS three times, and $200 \mu \mathrm{L}$ FCS free culture medium was added per well, which was kept at $37{ }^{\circ} \mathrm{C}$ in the incubator overnight. 


\section{Incubation of primary human hepatocytes with test compounds and cytotoxicity test}

The day after plating, the cells were exposed to the concentrations of test compounds as indicated in the "Results" section. If solubility was sufficient, the concentration range was adjusted to include at least one cytotoxic (below $\mathrm{EC}_{50}$ ) concentration. For some compounds, the used concentrations were limited by the maximal solubility of the corresponding compound. In case of water soluble compounds, substances were dissolved in medium and sterile filtered using $0.22-\mu \mathrm{m}$ membrane filters before adding to the cells. For compounds not sufficiently soluble in culture medium, DMSO was used as a solvent. If sufficient, $0.1 \%$ DMSO was used. Only when the cytotoxic test compound concentrations were not reached with $0.1 \%$ DMSO, the solvent concentration was increased to $0.5 \%$. The applied DMSO stock solutions for each compound and the tested compound concentrations are given in Suppl. Table 1A and B. The DMSO stock solutions were added to the culture medium to obtain the final test concentrations. The culture medium contains $11 \mathrm{mM}$ glucose. Therefore, the indicated concentrations of glucose were added additionally to this basal glucose concentration. For single exposure, the cells were incubated with compounds for $24 \mathrm{~h}$ or $48 \mathrm{~h}$; for repeated exposure, the compound-containing medium was renewed every $48 \mathrm{~h}$ and the cells were incubated for a total of 7 days. The cytotoxicity test (Cell-Titer-Blue) was performed according to an optimized SOP (Supplement 3) and the manufacturer's instructions. Briefly, after the cells were incubated with compounds for the indicated time periods, the compoundcontaining medium was removed, cells were washed with warm sterile PBS three times, and $100 \mu \mathrm{L}$ fresh FCS-free medium with $20 \%$ Cell-Titer-Blue $\AA$ reagent was added to each well. After $3 \mathrm{~h}$, the supernatant was transferred to black polystyrene 96-well plates and the fluorescence intensity was detected with the Tecan Infinite M200 Pro plate reader using the i-control software. Cells cultivated with culture medium only or with the solvent DMSO only $(0.1 \%$ and $0.5 \%$ DMSO; Suppl. Table 1) were used as a reference for $100 \%$ viability. The applied solvent concentrations of $0.1 \%$ or $0.5 \%$ DMSO did not cause any cytotoxicity compared to cells cultivated in medium without DMSO. Cell viability was calculated after background subtraction and expressed as percentage of control. PHH of three donors were used as three biological replicates and for each donor four technical replicates were analyzed for the fluorescence read out.

\section{Statistical analysis}

The raw data were initially processed as follows: background controls (fluorescence values from Cell-Titre-Blue Reagent ${ }^{\circledR}$ mixed with medium that was not in contact with cells) were subtracted from each data point. Replicates of control values and of exposed samples were averaged (donor wise). Next, averaged exposed samples were divided by the corresponding averaged control values and multiplied by 100 to obtain a percentage. Based on the assumption that the response dependency of the concentration can be described by a sigmoidal curve, a four-parameter log-logistic model (4pLL) was fitted to the data. Due to the non-linearity of the 4pLL model, the function was approximated according to the least square method with the Gauss-Newton algorithm. Then the individual values of each concentration were divided by the value of the left asymptote of the fitted curve. Again, a 4pLL model was fitted to the data. $\mathrm{EC}_{20}$ and $\mathrm{EC}_{50}$ values were calculated as the concentrations where the sigmoidal curve attains the values 80 and 50\%, respectively. The advantage of this fitting procedure is that the left asymptote is used as a control level for calculation of $\mathrm{EC}_{50}$ and $\mathrm{EC}_{20}$ values which are more robust than just using the values of the solvent controls. For calculating confidence intervals of the EC values, the concept of the ALEC (absolute lowest effective concentration) was used (Grinberg 2017). $\mathrm{EC}_{20}$ and $\mathrm{EC}_{50}$ values were calculated as the concentrations where the sigmoidal curve attains the values $80 \%$ and $50 \%$, respectively. Confidence intervals of the EC values were calculated using ALEC (absolute lowest effective concentration) and the delta method, as described in Grinberg 2017. EC values above the highest tested concentration, as well as for cases where the left asymptote lies above 80 or $50 \%$, respectively, were recorded as "> highest concentration". The EC values of the three donors were summarized as follows: the median was used when all three EC values were smaller than the highest tested concentration. When two of the three EC values were below the highest tested concentration, the second lowest value was used. When only one of the three EC values was below the highest tested concentration, values of the three donors were summarized as "> highest concentration". The latter values were only included as descriptive measures into the "Results" section but were excluded from further calculations, such as the establishment of the box plots in Fig. 2c and the correlation analyses in Figs. 3 and 4. For the test compound melatonin, day 1 , the value of the first donor was omitted and the mean of the two other values was used. Pearson correlation coefficients were calculated between the logarithmic EC summary values for two incubation times. An upper and lower threefold deviation line was added to the correlation plots. The upper deviation lines were obtained by adding three ( $\log 10$ scale) to the $Y$-axis distance of the regression line. Correspondingly, the lower deviation line was obtained by subtracting three ( $\log 10$ scale) from the $Y$-axis distance of the regression line. The statistical analyses were performed with the statistical programming language R-version 3.1.1. [https://www.R-project.org/]. For fitting concentration-response curves, the R-package drc 
(http://journals.plos.org/plosone/article?id=10.1371/journ al.pone.0146021) was used.

\section{Results}

\section{Selection of test compounds and concentration ranges}

In total, 30 compounds were selected for time- and concentration-dependent cytotoxicity testing (Suppl. Table 1). In addition to pharmaceuticals, ethanol, glucose, vitamin $\mathrm{C}$ and DMSO were also tested. For half of the selected compounds an increased risk of hepatotoxicity was previously reported, whereas no increased risk has been reported for the further 15 compounds at therapeutic doses (Suppl. Table 1). The tested concentrations and solvents are given in Suppl. Table 1.

\section{Influence of the incubation period on cytotoxicity}

For cytotoxicity testing, cryopreserved hepatocytes from three donors were used, whose characteristics are summarized in Table 1. Three representative examples of the cytotoxicity tests are given in Fig. 1, and all results and raw data are available in the supplement (Suppl. Fig. 1; Suppl. Table 2-4). The examples of propranolol (PPL), levofloxacin (LEV) and clonidine (CLON) illustrate a concentrationdependent increase in cytotoxicity for all three exposure periods (Fig. 1a-c). In Fig. 1, the $\mathrm{EC}_{50}$ is illustrated. Similarly, the $\mathrm{EC}_{20}$ values were determined (Suppl. Fig. 1).

To summarize the data, bar plots were generated for median $\mathrm{EC}_{50}$ values of all compounds (Fig. 2a). The figure illustrates the large, more than 1000-fold, differences in $\mathrm{EC}_{50}$ values among the compounds, with ketoconazole and promethazine representing the most, and dimethyl sulfoxide, glucose and ethanol the least cytotoxic compounds for all tested incubation periods. Next, the ratios of the median $\mathrm{EC}_{50}$ values for the different incubation periods were calculated (Fig. 2b). Acetaminophen is an example of a compound for which cytotoxicity did not increase after 7 days compared to 1 day of incubation (but rather showed a decrease).
In contrast, busulfan shows a relatively strong increase in cytotoxicity after 7 days compared to 1 day of incubation (Fig. 2b). The median $\mathrm{EC}_{50}$ ratio of all compounds was highest (1.89-fold) for days 1-7 (Fig. 2c). The second highest median $\mathrm{EC}_{50}$ ratio (1.78-fold) was obtained for days 1-2. Median values of $\mathrm{EC}_{50}$ ratios of all compounds at day 2 and day 7 did not show a major difference but for many individual compounds the ratio was different from one (Fig. 2b). The complete set of $\mathrm{EC}_{50}$ and $\mathrm{EC}_{20}$ values including the ratios is given in Suppl. Fig. 2 and Suppl. Table 2.

For statistical evaluation, correlation plots were created for $\log _{10} \mathrm{EC}_{50}$ values with the different incubation periods on the $x$-and $y$-axes. The longer incubation period is shown on the $y$-axis (Fig. 3). Data points indicate the experimentally obtained $\mathrm{EC}_{50}$ values for the 30 compounds, for which the median of three donors is shown. For some of the less toxic compounds, viability was not sufficiently reduced to calculate an $\mathrm{EC}_{50}$ value, because their limited solubility did not allow for the testing of higher concentrations. Toxicity data of compounds that did not reach $\mathrm{EC}_{50}$ or $\mathrm{EC}_{20}$ were not used for further calculations, such as box plot (Fig. 2c) or correlation analyses (Figs. 3, 4). EC $\mathrm{E}_{50}$ values after 1 and 7 days of incubation showed a high correlation with a correlation coefficient $(R)$ of 0.985 (with $95 \%$ confidence interval [0.960, 0.994]) (Fig. 3a). A high correlation coefficient means that the relationship between days 1 and 7 is similar across most compounds. If all compounds would lie on or very close to the regression line, this would mean that the $\mathrm{EC}_{50}$ values of longer incubation periods could be deduced from the $\mathrm{EC}_{50}$ values of shorter incubation periods. The majority of compounds were within a threefold deviation range from the regression line (plotted as a parallel line on logarithmic scale); however, the distance of individual compounds from the regression line may differ by more than fivefold (Fig. 3a). The compounds with the highest day 1/7 ratio are busulfan, famotidine and isoniazid. Similarly good correlations were obtained for the scatter plots of day 1 versus day $2(R=0.964)$ (Fig. 3b), as well as day 2 versus day 7 $(R=0.981)$ (Fig. 3c). The majority of data points were below the diagonal line, indicating higher levels of cytotoxicity for the longer incubation period. Similar correlation plots were obtained for both $\mathrm{EC}_{50}$ and $\mathrm{EC}_{20}$ (Fig. $4 \mathrm{a}-\mathrm{c}$ ). As observed

Table 1 Characteristics of the hepatocyte donors

\begin{tabular}{|c|c|c|c|c|c|c|c|c|}
\hline $\begin{array}{l}\text { Donor no. and } \\
\text { abbreviation }\end{array}$ & Sex & Age (year) & Diagnosis & Height $(\mathrm{cm})$ & Weight (kg) & Medication & $\begin{array}{l}\text { Alcohol } \\
(\mathrm{Y} / \mathrm{N})\end{array}$ & $\begin{array}{l}\text { Tobacco } \\
(\mathrm{Y} / \mathrm{N})\end{array}$ \\
\hline $1(\mathrm{AFJ})$ & M & 54 & Heart failure & 177.8 & 75 & Advil, Prilosec & $\mathrm{Y}$ & $\mathrm{Y}$ \\
\hline $2(\mathrm{IAN})$ & M & 48 & Head trauma & 177.8 & 70 & None & $\mathrm{Y}$ & $\mathrm{Y}$ \\
\hline 3 (MSW) & M & 69 & CVA, stroke & 179.8 & 80 & None & $\mathrm{Y}$ & $\mathrm{Y}$ \\
\hline
\end{tabular}

Detailed donor characteristics as provided by the commercial source of human hepatocytes (Supplement 1)

CVA cerebral vascular accident 
(A)

Day 1

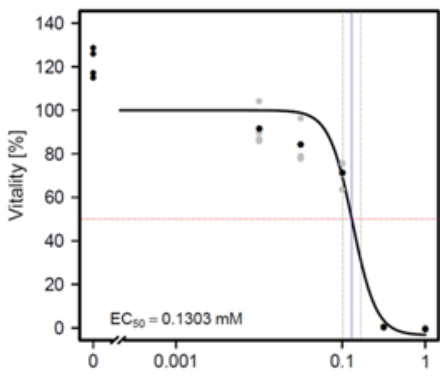

Concentration [mM]

Day 2

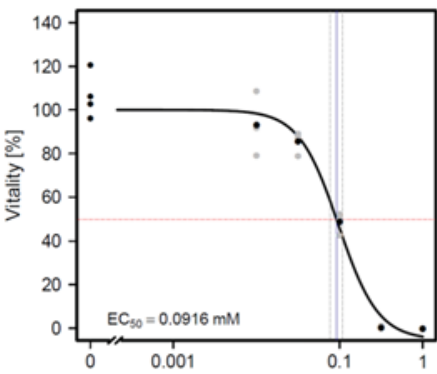

Concentration [mM]

Day 7

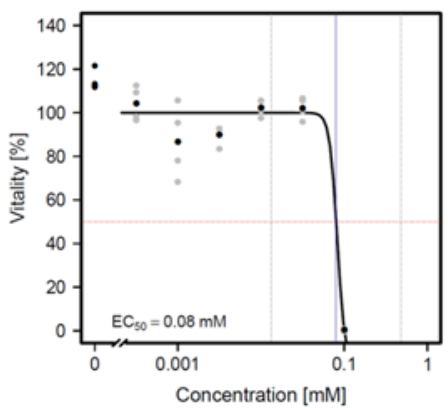

Donor 2
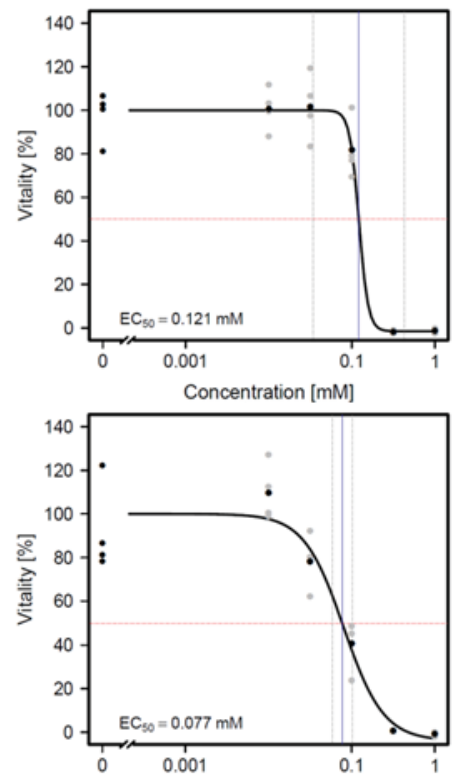

Concentration [mM]

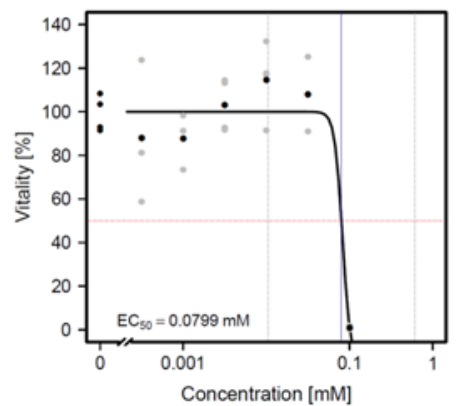

Donor 3
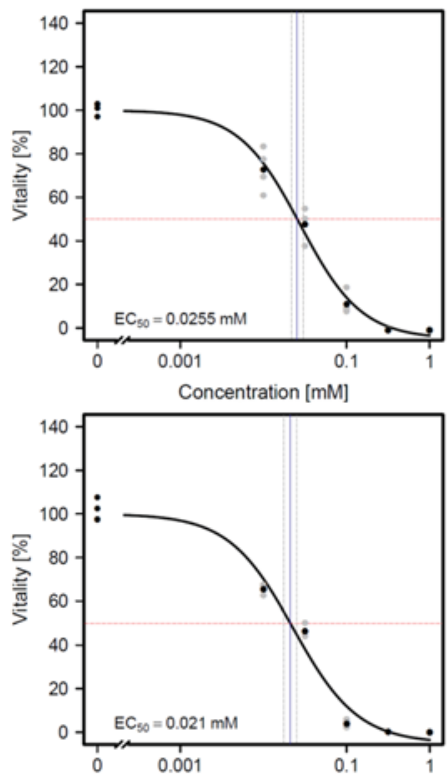

Concentration [mM]

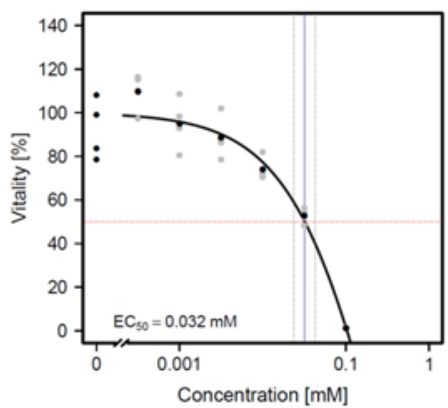

Propranolol

Fig. 1 Representative examples of Cell-Titer-Blue cytotoxicity data for propranolol (a), levofloxacin (b), and clonidine (c) in primary human hepatocytes after incubation for 1, 2 and 7 days. The corresponding data for all 30 compounds are available in Suppl. Fig. 1 and Suppl. Table 2-4. The concentration-dependent curves represent data from three donors with four technical replicates each. The cell viability for each concentration is presented as the percentage of untreated

for $\mathrm{EC}_{50}$, lower values were also obtained for $\mathrm{EC}_{20}$, when longer incubation periods were used. Correlation coefficients of the $\mathrm{EC}_{20}$ values (Fig. 3) were lower compared to those of $\mathrm{EC}_{20}$ (Fig. 4) suggesting that just beginning cytotoxicity $\left(\mathrm{EC}_{20}\right)$ shows larger deviations from the regression line than half-maximal $\left(\mathrm{EC}_{50}\right)$ cytotoxicity.

\section{Influence of the incubation period on cytotoxicity in published data}

Recently, Proctor et al. (2017) published cytotoxicity data with different incubation periods. To our knowledge, this is the only available public data set of human hepatocytes where cytotoxicity of a larger number of compounds has been studied with different exposure periods (data controls. Gray symbols indicate the viability values for each technical replicate normalized to untreated controls; whereas, black symbols represent the mean values of all technical replicates for each concentration. The vertical line indicates the concentration which causes $50 \%$ loss of viability $\left(\mathrm{EC}_{50}\right)$. The dashed vertical line shows the $95 \%$ confidence interval for the concentration. The resulting $\mathrm{EC}_{50}$ values are given for each panel. (Color figure online)

in supplement Table S4 of Proctor et al. 2017). Proctor et al. incubated spheroids of human hepatocytes for 4 and 14 days. However, they did not analyze correlations between the time points in their study. A correlation analysis was performed in the present study and is illustrated in Fig. 5. The result shows a highly significant correlation with a correlation coefficient of $R=0.894$, which is similar to the correlation coefficients obtained for our data. The largest difference between day 14 and day 5 was obtained for atorvastatin $\left(\mathrm{EC}_{50}\right.$ day 14: $0.006 \mathrm{mM} ; \mathrm{EC}_{50}$ day 5: $0.0707 \mathrm{mM}$ ). Surprisingly, for some compounds lower $\mathrm{EC}_{50}$ values were obtained for the shorter incubation period (e.g. flutamide: $\mathrm{EC}_{50}$ day 14: $0.0257 \mathrm{mM} ; \mathrm{EC}_{50}$ day 5: $0.0125 \mathrm{mM}$; and phenformin: $\mathrm{EC}_{50}$ day 14: $0.0125 \mathrm{mM}$; $\mathrm{EC}_{50}$ days 5-6: $0.0077 \mathrm{mM}$ ). 
(B)

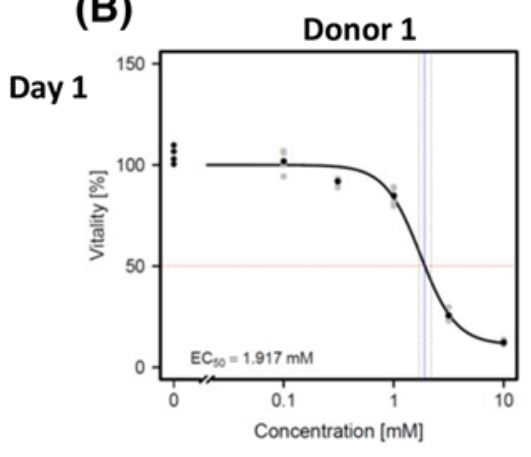

Day 2
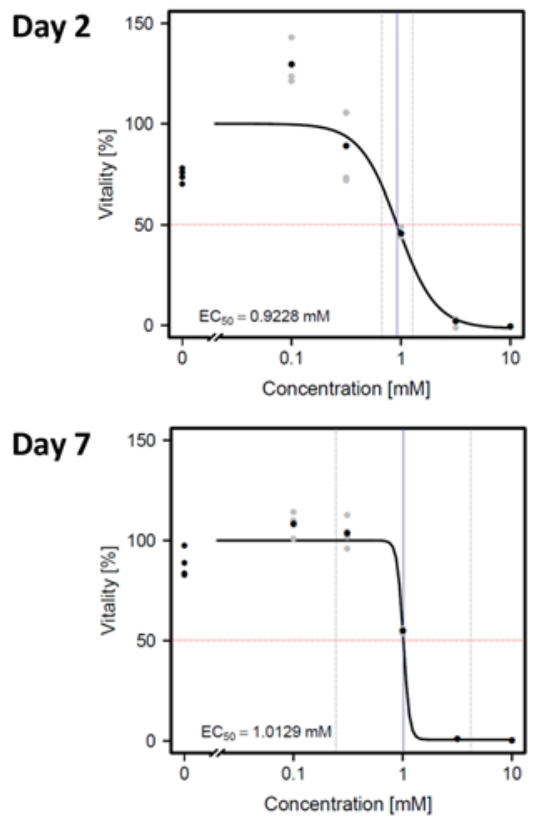

Donor 2
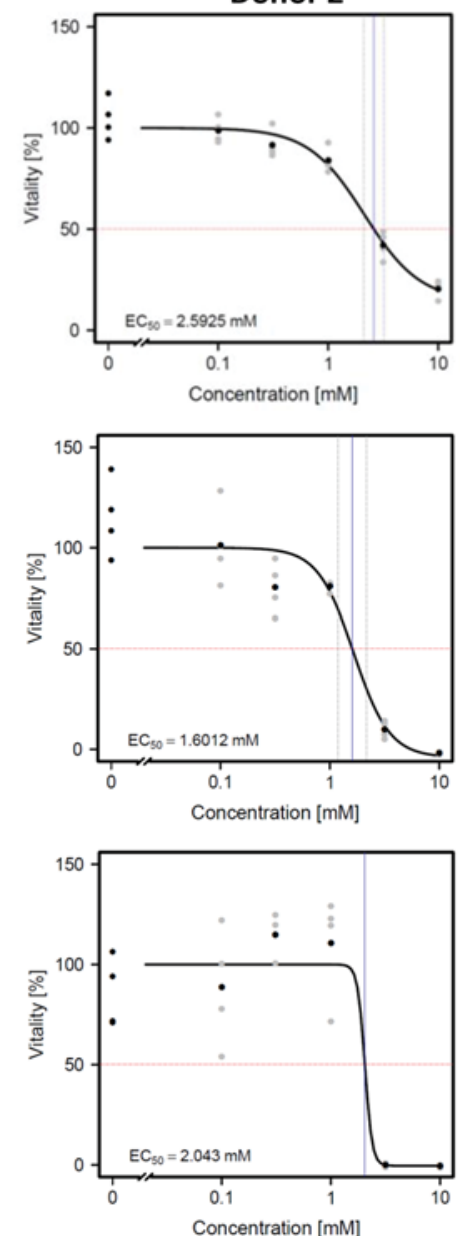

Donor 3

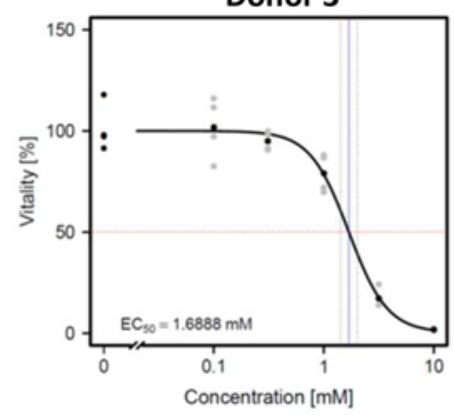

Levofloxacin

Fig. 1 (continued)

\section{Discussion}

In the present study, the influence of the incubation period on cytotoxicity of 30 compounds was studied in cultivated primary human hepatocytes. This research question is of relevance, because a specific incubation period has to be chosen for in vitro testing. Currently, large efforts are undertaken to establish in vitro tests with the long-term goal to predict human toxicity (Daneshian et al. 2016; Frey et al. 2014; Ghallab 2015; Kampe et al. 2014; Kim et al. 2015; Leist et al. 2017). For this purpose, in vitro systems with human (Deharde et al. 2016; Godoy et al. 2013, 2015, 2016; Grinberg et al. 2014; Luckert et al. 2017; Pfeiffer et al. 2015) as well as rodent (Arbo et al. 2016; Hengstler et al. 2000; Hewitt et al. 2007; Reif et al. 2015) hepatocytes, neuronal cells (Colaianna et al. 2017; Krug et al. 2013; Rempel et al. 2015; Shinde et al. 2017; Waldmann et al. 2014), kidney cell lines (Gong et al. 2016; Liu et al. 2016) and cardiomyocytes (Chaudhari et al. 2016; Gaspar et al. 2014) have been optimized. One challenge of these in vitro approaches is the choice of adequate exposure periods with test compounds. For practical reasons, short-exposure periods such as $24 \mathrm{~h}$ or $48 \mathrm{~h}$ are preferred. However, it has been discussed, whether longer incubation periods, such as 14 days or even months, may be superior to predict repeated dose toxicity (Proctor et al. 2017). Moreover, washout experiments with an initial exposure period with test compounds followed by test compound-free incubation or repeated exposure and washout periods may be considered. The idea behind these more complex in vitro exposure protocols is to imitate features of human pharmacokinetics. However, it should be considered that an in vitro system may respond differently to repeated or chronic exposure than an organ in vivo. A suitable example of this is liver cirrhosis where repeated exposure to hepatotoxic compounds leads to hepatocyte death followed by stellate cell activation and secretion of extracellular matrix (Hammad et al. 2017; Jansen et al. 2017). However, since the currently available in vitro systems do not recapitulate 
(C)
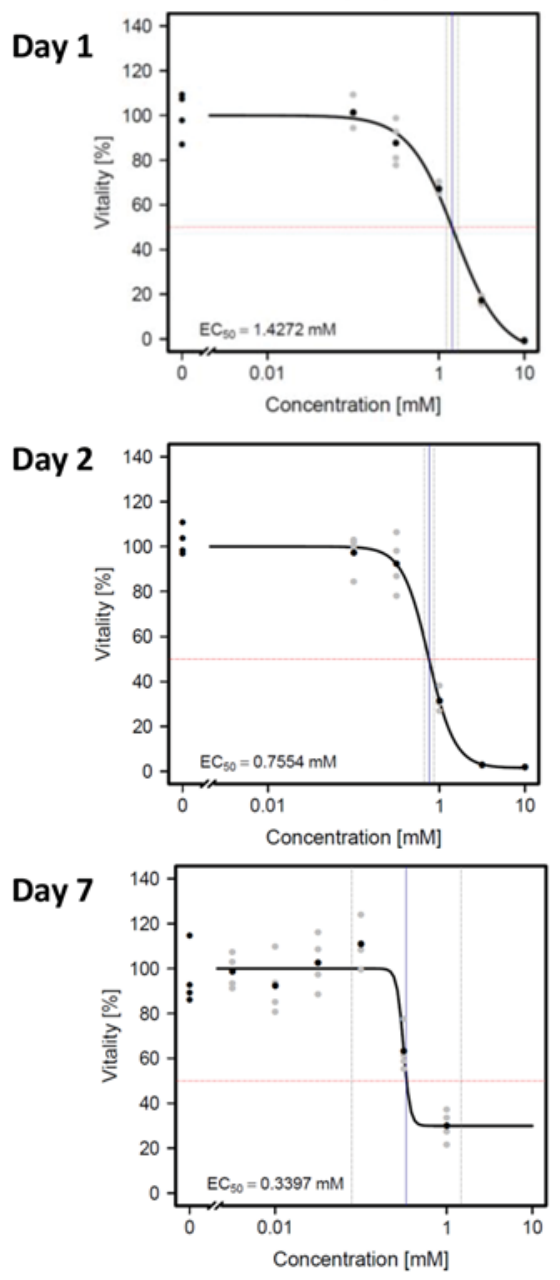

Donor 2
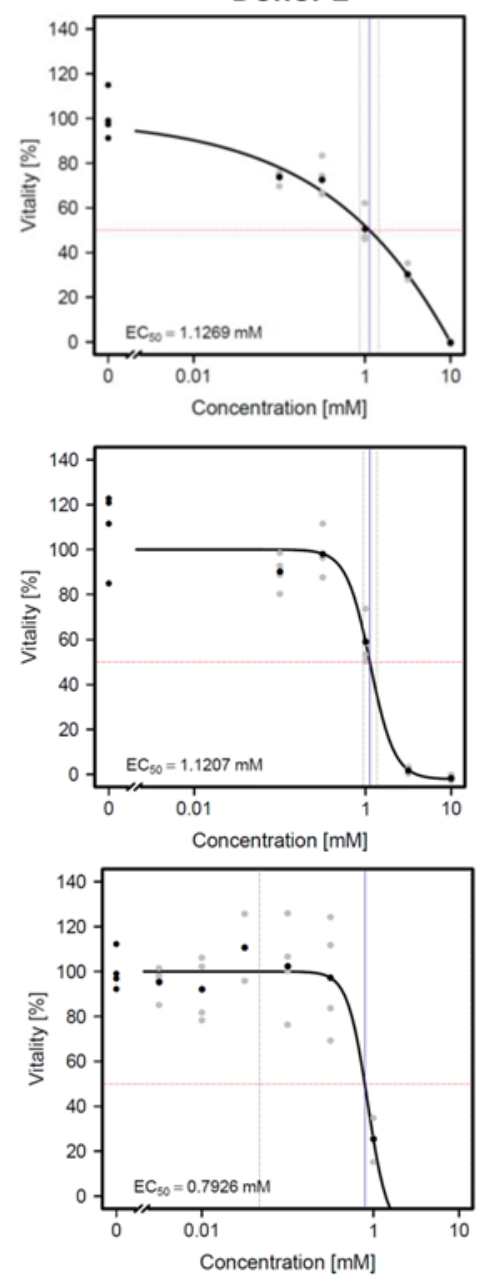

Donor 3

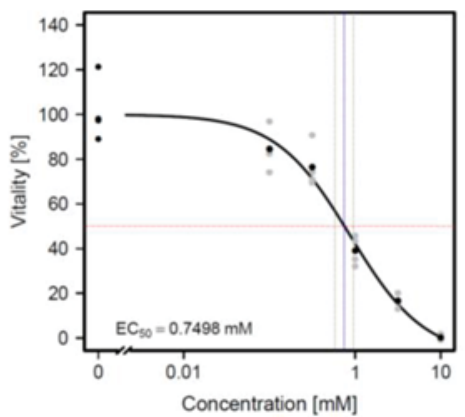

Clonidine
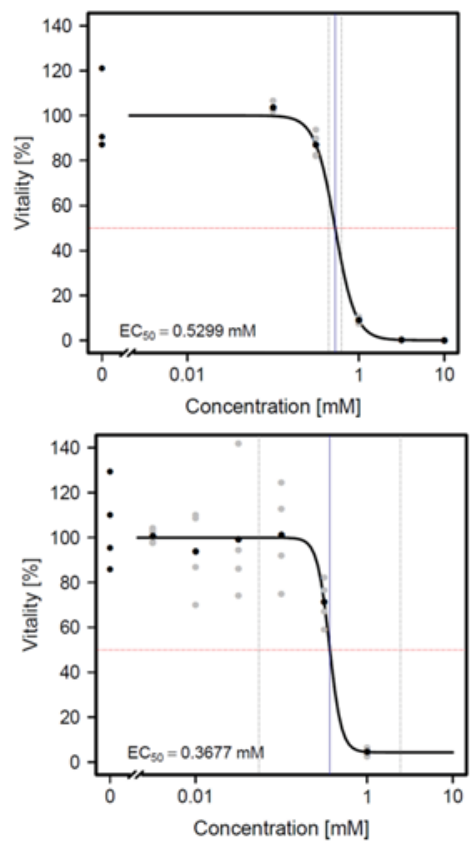

Fig. 1 (continued)

the physiological interactions of hepatocytes, sinusoidal endothelial cells and stellate cells (Godoy et al. 2013; Hoehme et al. 2010, 2017), it remains challenging to study the full process of pathogenesis. On the other hand, the single doses of hepatotoxic compounds (e.g. $\mathrm{CCl}_{4}$ ) that are typically used in repeated dose studies to induce fibrosis in mice or rats are already high enough to cause hepatocyte death within hours (Ghallab et al. 2016; Schliess et al. 2014). Therefore, the hepatocyte in vitro system is not able to recapitulate the complex adverse outcome, such as fibrosis; it will at best correctly recapitulate one key event, namely hepatocyte death.

Currently, relatively little is known regarding the degree to which incubation period influences $\mathrm{EC}_{20}$ or $\mathrm{EC}_{50}$ values of cultivated hepatocytes. Recently, Proctor et al. (2017) compared cytotoxicity of liver spheroids incubated for 5 and 14 days. However, it is unknown how their results obtained with these relatively long-incubation periods compared to shorter exposure periods (e.g. 1 or 2 days).
Therefore, the present study was performed to compare cytotoxicity of human hepatocytes after 1,2 and 7 days of test compound exposure using 30 test compounds. A major goal was to understand whether the $\mathrm{EC}_{50}$ changes by a similar factor for all compounds, when longer exposure periods are chosen, or whether large compound specific differences occur. The results of the present study demonstrated a higher degree of cytotoxicity for an incubation period of 7 days compared to 1 or 2 days. The results $\left(\mathrm{EC}_{50}\right.$ or $\left.\mathrm{EC}_{20}\right)$ obtained after 7 days of incubation showed an excellent correlation with the data after 1 or 2 days, with correlation coefficients higher than 0.9. However, the ratios in $\mathrm{EC}_{50}$ values after 7 days compared to day 2 or day 1 differed among the 30 compounds, although these differences remained within one order of magnitude. Compounds with a relatively strong decrease in $\mathrm{EC}_{50}$ after 7 days compared to 1 day are busulfan, famotidine, and isoniazid (Fig. 2b). The reasons, why these compounds require more time to exert their cytotoxic effect, remain to be studied. For 

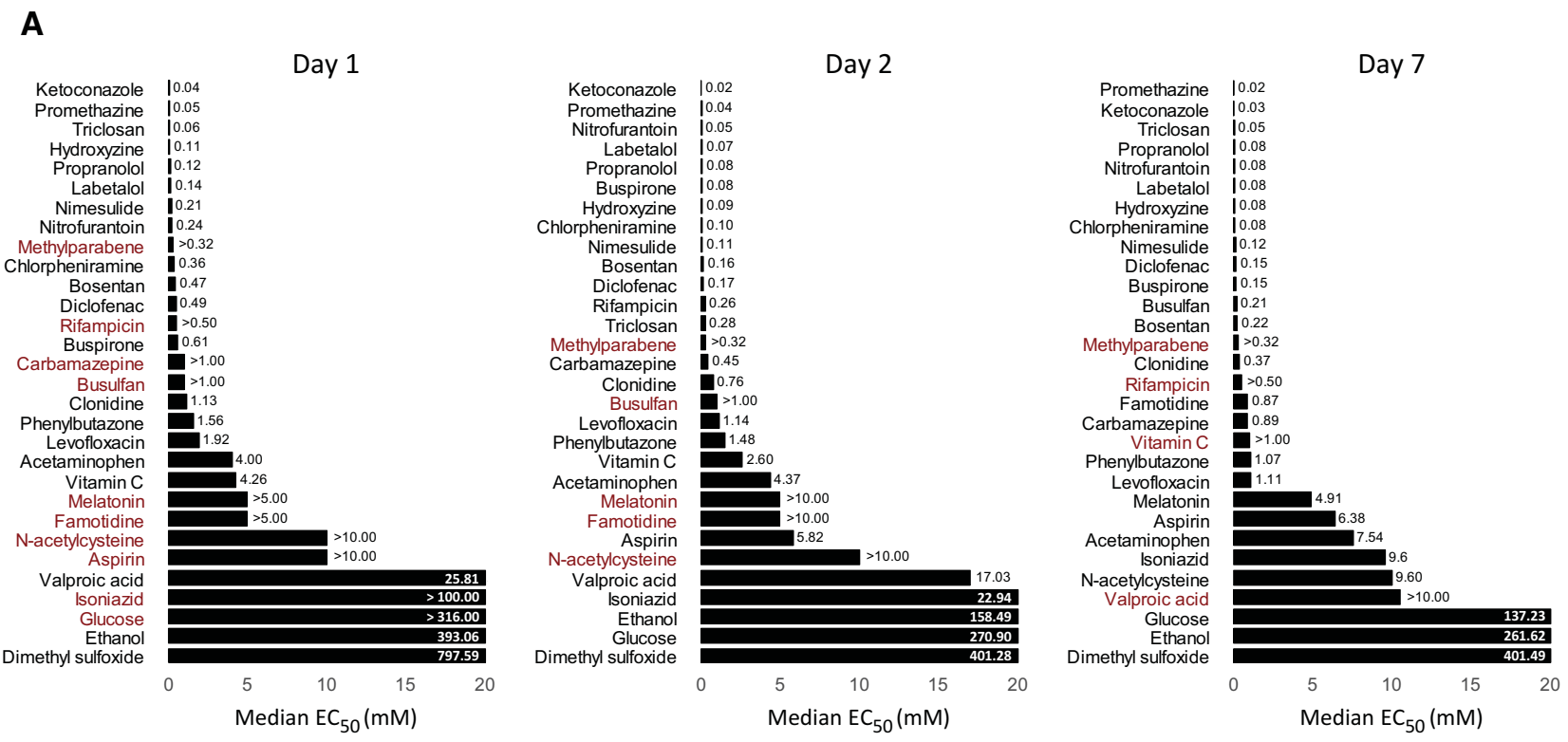

B

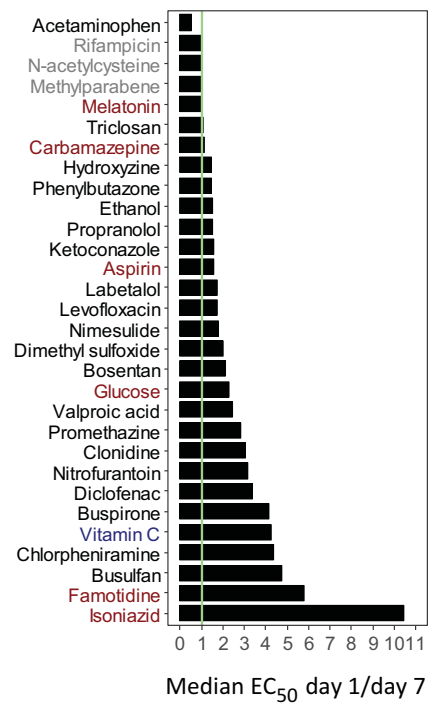

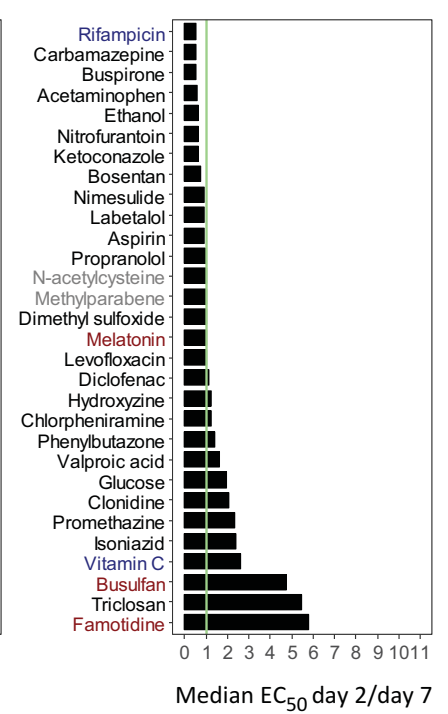

C

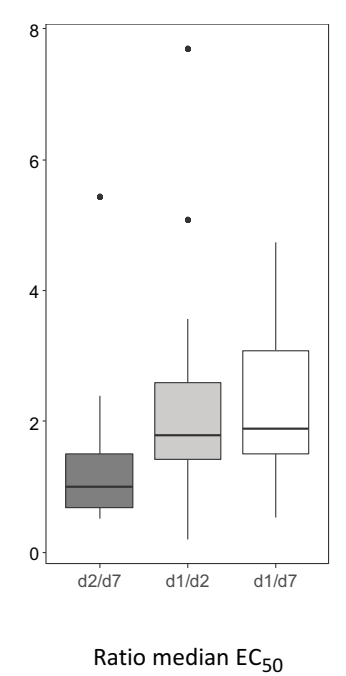

Fig. 2 Time dependency of cytotoxicity. a $\mathrm{EC}_{50}$ values of all test compounds after incubation of cultivated human hepatocytes for 1,2 and 7 days. Data are median values of three donors. The raw data are available in Suppl. Tables 2 and 3. Substance labeled in black represents compounds where the $\mathrm{EC}_{50}$ values could be determined. Substances labeled in red are those where the $\mathrm{EC}_{50}$ could not reached at the highest tested concentration due to limited solubility. $\mathbf{b}$ Ratios of $\mathrm{EC}_{50}$ values on day 1 and day 7 , day 1 and day 2, as well as day 2 and day 7 . The higher the ratio the more cytotoxic the effect will be at longer incubation periods. Substances labeled in black are those

busulfan, a non-specific alkylating antineoplastic agent, the time required between DNA alkylation and cytotoxicity may be relatively long, because cultivated human hepatocytes do not proliferate. In contrast, acetaminophen shows where $\mathrm{EC}_{50}$ values could be determined for both incubation periods. Red color indicates substances where the different colors of substance names indicate that the $\mathrm{EC}_{50}$ was not reached (i) at the shorter incubation period (red), (ii) at the longer incubation period (blue), and (iii) at both incubation periods (gray) due to limited solubility. c Box plot presentation of the $\mathrm{EC}_{50}$ ratios of all compounds. The ratio on the $y$-axis gives the increase in cytotoxicity of 7 compared to 2 days, 2 days compared to 1 day, and 7 days compared to 1 day. For the box plot analysis, only those compounds were considered for which $\mathrm{EC}_{50}$ values were reached. (Color figure online)

only relatively little increase in cytotoxicity upon comparing 1 and 7 days of incubation. Possibly, the mechanism of acetaminophen toxicity with regard to its metabolism to the reactive $N$-acetyl- $p$-benzoquinone imine (NAPQI), 
Fig. 3 Correlation plots for cytotoxicity $\left(\mathrm{EC}_{50}\right)$ values in primary human hepatocytes with the incubation periods given on the $X$ and $Y$-axes. Each data point represents a median value of three biological replicates (three donors). Black data points indicate the experimentally obtained $\mathrm{EC}_{50}$ values. Compounds for which viability was not sufficiently reduced to allow calculation of an $\mathrm{EC}_{50}$ were not included into this analysis. a Correlation plot between $\mathrm{EC}_{50}$ values after 1 versus 7 days of test compound incubation; 1 versus 2 days (b) and 2 versus 7 days (c) of test compound exposure. $R$ : correlation coefficient; $P: Y$ : linear equation

protein adduct formation, and mitochondrial toxicity represents a relatively fast process.

Comparing the cytotoxic effect after 1, 2 and 7 days incubation periods shows a relatively strong increase in cytotoxicity between 1 and 7 days, and between 1 and 2 days; however, the increase between day 2 and day 7 is relatively small (Fig. 2c). Therefore, 2 days may represent an adequate choice for cytotoxicity tests with human hepatocytes in future studies, offering the practical advantage that less culture medium changes are required.

To our knowledge, the only publicly available data set of cultivated human hepatocytes with different incubation periods has recently been made available (Proctor et al. 2017). These authors cultivated human hepatocytes as spheroids, therefore, using a different cultivation system than in the present study. Nevertheless, the data of Proctor et al. are of particular interest for the present study, because they compared cytotoxicity after 14 days of test compound exposure to 5-day long-incubation periods. A strong correlation was observed between $\mathrm{EC}_{50}$ values after 5 and 14 days of test compound exposure with a comparable high correlation coefficient as in the data generated in the present study. Surprisingly, the data of Proctor et al. (2017) showed higher cytotoxicity for two compounds with the shorter incubation period. These compounds are flutamide $\left(\mathrm{EC}_{50}, 14\right.$ days: $0.0257 \mathrm{mM}$; $\mathrm{EC}_{50}, 5$ days: $0.0125 \mathrm{mM}$ ) and phenformin $\left(\mathrm{EC}_{50}, 14\right.$ days: $0.0125 \mathrm{mM}$; $\mathrm{EC}_{50}, 5$ days: $\left.0.0077 \mathrm{mM}\right)$. Similar scenarios where lower $\mathrm{EC}_{50}$ values for shorter compared to the longer incubation periods were also observed for a few compounds in the present study. However, these results should be interpreted with caution and further reproduction is required before possible biological explanations are discussed.

Calculation of $\mathrm{EC}_{50}$ and $\mathrm{EC}_{20}$ values in the present study was based on the assumption that the concentration-response relationship can be described by a sigmoidal curve. Therefore, a four-parameter log-logistic model (4pLL) was fitted to the cytotoxicity data. This approach reduces the influence of outliers at individual concentrations. Repeat experiments performed with the same batch of cryopreserved hepatocytes demonstrated that such deviations at individual concentrations are indeed technical outliers and not reproducible, which justifies the use of the 4pLL model.
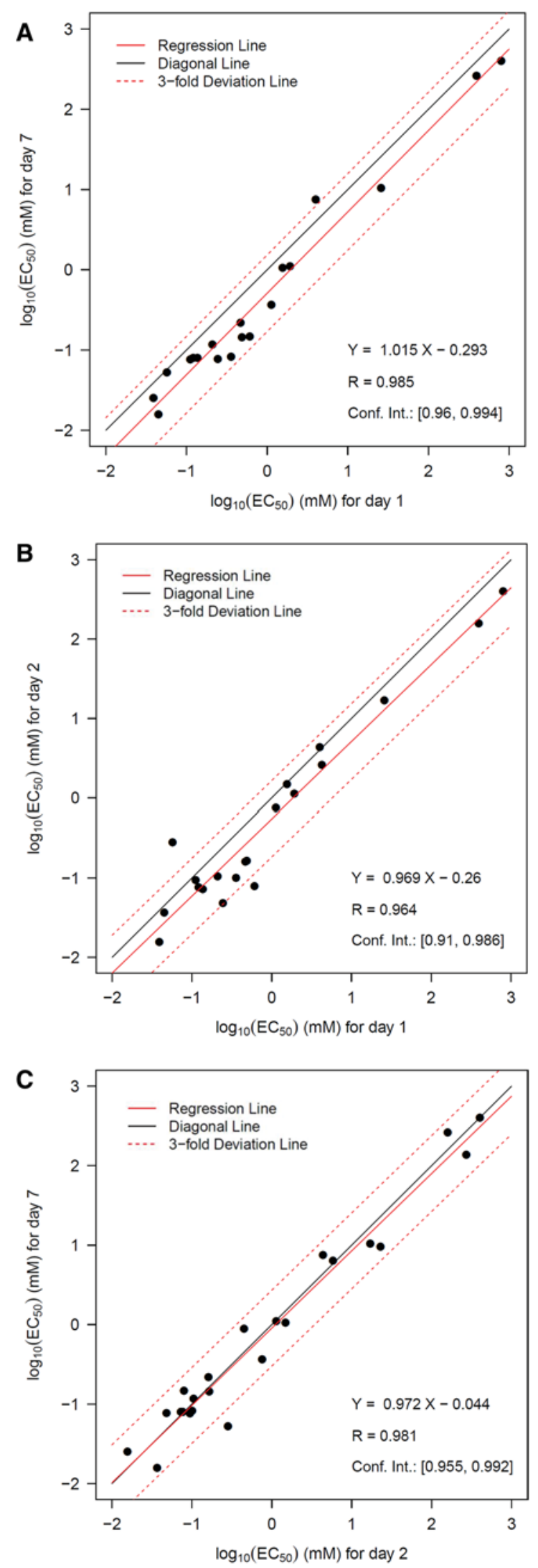

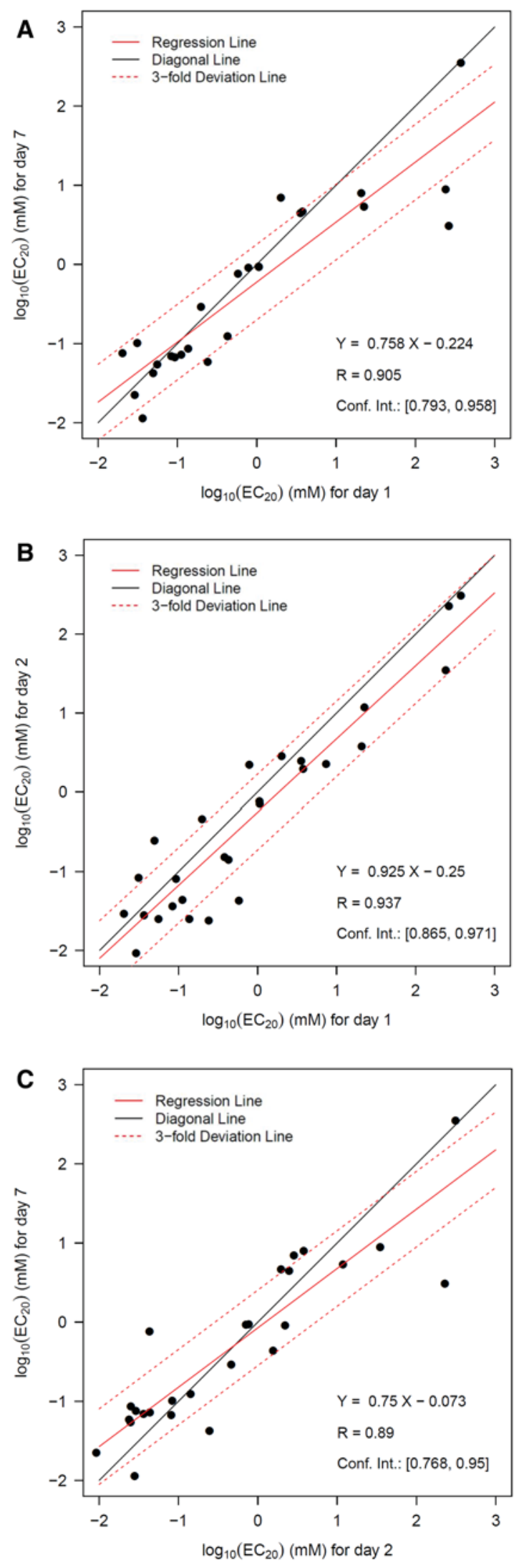

Fig. 4 Correlation plots for $\mathrm{EC}_{20}$ cytotoxicity data gene as described in Fig. 3. However, the $\mathrm{EC}_{20}$ values of cytotoxicity are shown

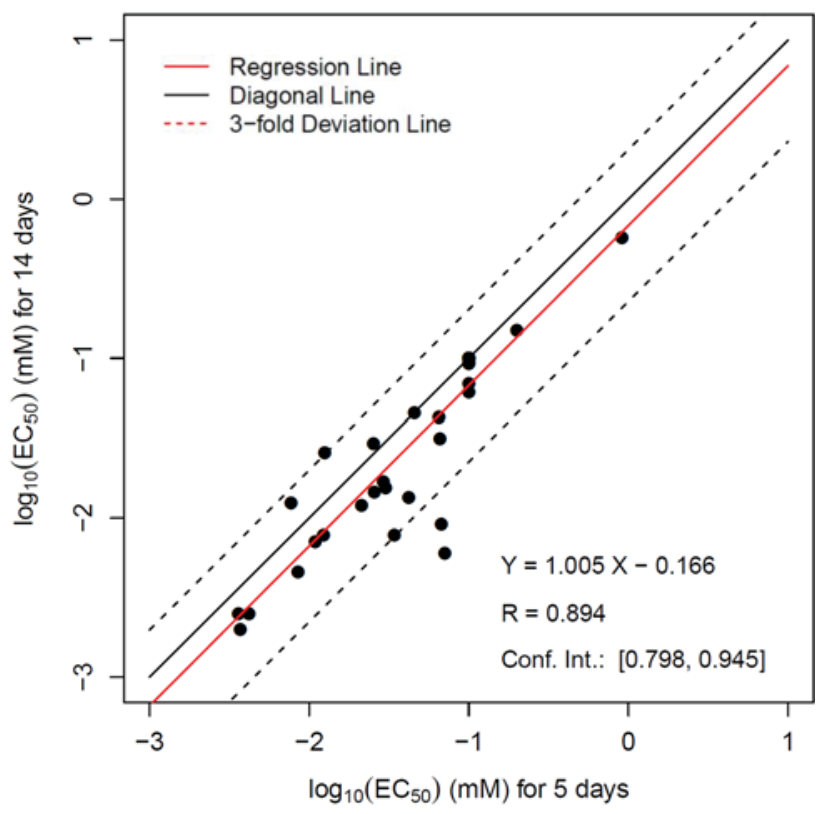

Fig. 5 Correlation plots for cytotoxicity $\left(\mathrm{EC}_{50}\right)$ values in spheroids of primary human hepatocytes with the short-term (5 days) and longterm (14 days) incubation periods given on the $X$ and $Y$-axes. The plots were generated from published data as presented in supplement Table S4 of Proctor et al. (2017)

In conclusion, for longer incubation periods, such as 7 days compared to 1 day, cytotoxicity usually increases. However, there is an excellent correlation of $\mathrm{EC}_{50}$ and $\mathrm{EC}_{20}$ values obtained for the different incubation periods. Whether cytotoxicity data obtained for longer incubation periods correspond better to the in vivo situation still remains to be determined.

Acknowledgements The study was supported by the European Union's Horizon 2020 research and innovation programme (EUToxRisk; no. 681002) with additional contributions of the projects StemCellNet (BMBF, 01EK1604A), Liver Simulator (BMBF, 031A355A), DILI (BMBF, 031L0074F), LiSyM (BMBF, 031Loo45), LivSysTransfer (BMBF, 0101-31Q0517), InnoSysTox (BMBF/EU, 031L0021A), SteatoTox (031L0114B) and DEEP (BMBF, 01KU1216).

\section{Compliance with ethical standards}

Conflict of interest There is no conflict of interest.

\section{References}

Arbo MD, Melega S, Stober R et al (2016) Hepatotoxicity of piperazine designer drugs: up-regulation of key enzymes of cholesterol and lipid biosynthesis. Arch Toxicol 90(12):3045-3060

Bernal W, Auzinger G, Dhawan A, Wendon J (2010) Acute liver failure. Lancet 376(9736):190-201

Chaudhari U, Nemade H, Gaspar JA, Hescheler J, Hengstler JG, Sachinidis A (2016) MicroRNAs as early toxicity signatures of 
doxorubicin in human-induced pluripotent stem cell-derived cardiomyocytes. Arch Toxicol 90(12):3087-3098

Colaianna M, Ilmjarv S, Peterson H et al (2017) Fingerprinting of neurotoxic compounds using a mouse embryonic stem cell dual luminescence reporter assay. Arch Toxicol 91(1):365-391

Daneshian M, Kamp H, Hengstler J, Leist M, van de Water B (2016) Highlight report: launch of a large integrated European in vitro toxicology project: EU-ToxRisk. Arch Toxicol 90(5):1021-1024

Deharde D, Schneider C, Hiller T et al (2016) Bile canaliculi formation and biliary transport in 3D sandwich-cultured hepatocytes in dependence of the extracellular matrix composition. Arch Toxicol 90(10):2497-2511

Frey O, Misun PM, Fluri DA, Hengstler JG, Hierlemann A (2014) Reconfigurable microfluidic hanging drop network for multi-tissue interaction and analysis. Nat Commun 5:4250

Gaspar JA, Doss MX, Hengstler JG, Cadenas C, Hescheler J, Sachinidis A (2014) Unique metabolic features of stem cells, cardiomyocytes, and their progenitors. Circ Res 114(8):1346-1360

Ghallab A (2015) Interspecies extrapolation by physiologically based pharmacokinetic modeling. EXCLI J 14:1261-1263

Ghallab A, Celliere G, Henkel SG et al (2016) Model-guided identification of a therapeutic strategy to reduce hyperammonemia in liver diseases. J Hepatol 64(4):860-871

Godoy P, Hewitt NJ, Albrecht U et al (2013) Recent advances in 2D and $3 \mathrm{D}$ in vitro systems using primary hepatocytes, alternative hepatocyte sources and non-parenchymal liver cells and their use in investigating mechanisms of hepatotoxicity, cell signaling and ADME. Arch Toxicol 87(8):1315-1530

Godoy P, Schmidt-Heck W, Natarajan K et al (2015) Gene networks and transcription factor motifs defining the differentiation of stem cells into hepatocyte-like cells. J Hepatol 63(4):934-942

Godoy P, Widera A, Schmidt-Heck W et al (2016) Gene network activity in cultivated primary hepatocytes is highly similar to diseased mammalian liver tissue. Arch Toxicol 90(10):2513-2529

Gong X, Ivanov VN, Hei TK (2016) 2,3,5,6-Tetramethylpyrazine (TMP) down-regulated arsenic-induced heme oxygenase-1 and ARS2 expression by inhibiting Nrf2, NF-kappaB, AP-1 and MAPK pathways in human proximal tubular cells. Arch Toxicol 90(9):2187-2200

Grinberg M (2017) Statistical analysis of concentration-dependent, high-dimensional gene expression data. $\mathrm{PhD}$ thesis, University of Dortmund, 2017

Grinberg M, Stober RM, Edlund K et al (2014) Toxicogenomics directory of chemically exposed human hepatocytes. Arch Toxicol 88(12):2261-2287

Hammad S, Braeuning A, Meyer C, Mohamed F, Hengstler JG, Dooley $S$ (2017) A frequent misinterpretation in current research on liver fibrosis: the vessel in the center of CCl4-induced pseudolobules is a portal vein. Arch Toxicol 91(11):3689-3692

Hengstler JG, Utesch D, Steinberg P et al (2000) Cryopreserved primary hepatocytes as a constantly available in vitro model for the evaluation of human and animal drug metabolism and enzyme induction. Drug Metab Rev 32(1):81-118

Hewitt NJ, Lechon MJ, Houston JB et al (2007) Primary hepatocytes: current understanding of the regulation of metabolic enzymes and transporter proteins, and pharmaceutical practice for the use of hepatocytes in metabolism, enzyme induction, transporter, clearance, and hepatotoxicity studies. Drug Metab Rev 39(1):159-234

Hoehme S, Brulport M, Bauer A et al (2010) Prediction and validation of cell alignment along microvessels as order principle to restore tissue architecture in liver regeneration. Proc Natl Acad Sci USA 107(23):10371-10376

Hoehme S, Friebel A, Hammad S, Drasdo D, Hengstler JG (2017) Creation of three-dimensional liver tissue models from experimental images for systems medicine. Methods Mol Biol 1506:319-362

Jansen PL, Ghallab A, Vartak N et al (2017) The ascending pathophysiology of cholestatic liver disease. Hepatology 65(2):722-738

Kampe T, Konig A, Schroeder H, Hengstler JG, Niemeyer CM (2014) Modular microfluidic system for emulation of human phase I/ phase II metabolism. Anal Chem 86(6):3068-3074

Kim JY, Fluri DA, Marchan R et al (2015) 3D spherical microtissues and microfluidic technology for multi-tissue experiments and analysis. J Biotechnol 205:24-35

Krug AK, Kolde R, Gaspar JA et al (2013) Human embryonic stem cell-derived test systems for developmental neurotoxicity: a transcriptomics approach. Arch Toxicol 87(1):123-143

LeCluyse EL (2001) Human hepatocyte culture systems for the in vitro evaluation of cytochrome P450 expression and regulation. Eur J Pharm Sci 13(4):343-368

Leist M, Ghallab A, Graepel R et al (2017) Adverse outcome pathways: opportunities, limitations and open questions. Arch Toxicol 91(11):3477-3505

Liu G, Wang ZK, Wang ZY, Yang DB, Liu ZP, Wang L (2016) Mitochondrial permeability transition and its regulatory components are implicated in apoptosis of primary cultures of rat proximal tubular cells exposed to lead. Arch Toxicol 90(5):1193-1209

Luckert C, Schulz C, Lehmann N et al (2017) Comparative analysis of 3D culture methods on human HepG2 cells. Arch Toxicol 91(1):393-406

Ostapowicz G, Fontana RJ, Schiodt FV et al (2002) Results of a prospective study of acute liver failure at 17 tertiary care centers in the United States. Ann Intern Med 137(12):947-954

Pfeiffer E, Kegel V, Zeilinger K et al (2015) Featured article: Isolation, characterization, and cultivation of human hepatocytes and non-parenchymal liver cells. Exp Biol Med (Maywood NJ) 240(5):645-656

Proctor WR, Foster AJ, Vogt J et al (2017) Utility of spherical human liver microtissues for prediction of clinical drug-induced liver injury. Arch Toxicol 91(8):2849-2863

Reif R, Karlsson J, Gunther G et al (2015) Bile canalicular dynamics in hepatocyte sandwich cultures. Arch Toxicol 89(10):1861-1870

Rempel E, Hoelting L, Waldmann T et al (2015) A transcriptome-based classifier to identify developmental toxicants by stem cell testing: design, validation and optimization for histone deacetylase inhibitors. Arch Toxicol 89(9):1599-1618

Schliess F, Hoehme S, Henkel SG et al (2014) Integrated metabolic spatial-temporal model for the prediction of ammonia detoxification during liver damage and regeneration. Hepatology 60(6):2040-2051

Shinde V, Hoelting L, Srinivasan SP et al (2017) Definition of transcriptome-based indices for quantitative characterization of chemically disturbed stem cell development: introduction of the STOPToxukn and STOP-Toxukk tests. Arch Toxicol 91(2):839-864

Waldmann T, Rempel E, Balmer NV et al (2014) Design principles of concentration-dependent transcriptome deviations in drug-exposed differentiating stem cells. Chem Res Toxicol 27(3):408-420

Wilke RA, Lin DW, Roden DM et al (2007) Identifying genetic risk factors for serious adverse drug reactions: current progress and challenges. Nat Rev Drug Discov 6(11):904-916 